\section{Asymptomatischer polysomnographischer Befund}

Helga Peter ${ }^{1}$ und Thomas Penzel ${ }^{2}$

${ }^{1}$ Marburg, Deutschland

${ }^{2}$ Interdisziplinäres Schlafmedizinisches Zentrum, Charité -

Universitätsmedizin Berlin, Berlin, Deutschland

\section{Englischer Begriff}

asymptomatic polysomnographic finding

\section{Definition}

Positiver Befund in der $\downarrow$ „Kardiorespiratorischen Polysomnographie" bei einem bezüglich der schlafmedizinischen Anamnese beschwerdefreien Patienten. 